\title{
A Bandwidth Assignment Technique (Optimizing Technique) For Reliable Microwave Backhaul
}

\author{
${ }^{1}$ Vandana, ${ }^{2}$ Dr.vijay lamba \\ ${ }^{1}$ M.Tech ECE, HCTM, Kaithal ${ }^{2}$ Professor, HCTM, Kaithal
}

\begin{abstract}
First, the radio frequency spectrum is a limited natural resource which has been regulated worldwide to promote its efficient use. Second, the radio channel is a random and difficult communication medium. Actually, environment conditions can introduce instantaneously variations into the communication channel, likely leading to outage events. Therefore, capacity planning for wireless microwave backhaul requires additional reliability investigation.The capacity of microwave links is basically determined by the channel bandwidth and the modulation scheme used to transmit data. On the one hand, the assigned bandwidth for each link is a network engineer's decision subject to obtaining licenses upon payment of renewal fees whose values is usually in accordance with the amount of spectrum (e.g., in MHz) with which a license is associated. The amount of spectrum is generally available in modules, resulting in discontinuous step increasing cost functions on the links.
\end{abstract}

Keyword: chance constrained mathematical approach,an equilent ILP formulation,cutset based valid enqualities.

\section{Introduction}

In this paper, we investigate on conceiving reliable microwave backhaul networks under outage probability constraints. We introduce a joint optimization of data routing and bandwidth assignment that minimizes the total renewal fees of licenses, while handling all the traffic requirements simultaneously with a prescribed reliability level. It can be seen as a special case of the minimum cost multicommodity flow (MCMCF) with discontinuous step increasing cost functions on the links. In addition, we must consider probabilistic constraints to deal with random parameters (viz., modulation schemes) that impact the capacity of microwave links.Microwave backhaul solutions, although having limited bandwidth and suffering channel impairments, must degrade gracefully as environment conditions degrade. As a common practice, backhaul operators highly overprovision bandwidth during capacity planning to avoid traffic bottlenecks under adverse scenarios (when the performance of some links deteriorates). This approach, however, incurs additional investments that do not result in resource- and cost-efficient networks, besides leading to the inefficient use of the radio spectrum. Therefore, establishing better wireless backhaul solutions is not just a matter of adding bandwidth, but it also entails a complex decision aiming at enhancing network reliability to cope with channel fluctuations.

We then propose a (joint) chance-constrained mathematical programming approach to tackle this problem. Chance-constrained programming aims at finding the best solution remaining feasible for a given infeasibility probability tolerance. This approach is still considered as very difficult and widely intractable since the feasible region defined by a probabilistic constraint generally is not convex. In addition, among the vast literature on chance-constrained programming, few research work has been carried out to tackle combinatorial problems. Given these difficulties, we derive an equivalent ILP formulation for the case where the outage probabilities of the microwave links are independent and propose cutset-based valid inequalities to obtain strengthened formulations for this problem.

The remainder of this chapter is organized as follows. In Section 3.1, we present the context and motivation of this study. In Section 3.2, we give a brief introduction to chance-constrained programming. In Section 3.3, we introduce exact formulations for the application considered here. Section 3.4 is devoted to cutsetbased valid inequalities to improve these formulations. In Section 3.5, we discuss preliminary computational results illustrating the price of reliability and present a comparative study on the performance of the different formulations. In Section 3.6, some final remarks and comments on future work conclude the chapter.

\section{Chance Constrained Programming}

It appears as a specific model of stochastic optimization for dealing with random parameters in optimization problems. These random parameters take part of the inequilities and sometimes of the objectives function as well, describing the proper working of the system under consideration.To enhance the performance 
of ILP solvers several valid inequalities have been introduced called cutset based inequalities.). This can be expressed in terms of chance constraints (or probabilistic constraints). The constraint

$$
P(h j(x, z) \geq 0) \geq 1-£, j € J
$$

are called separate chance constraints, while the constraint

$$
P\left(\operatorname{hj}(\mathrm{x}, z) \_0, \mathrm{j} \in \mathrm{J}\right) \geq 1-£
$$

is called a joint chance constraint, where

$\mathrm{P}=$ probability measure

$\mathrm{x}=$ vector of decision variables

$z=$ vector of random parameters

$\mathrm{h}(\mathrm{x}, z) \geq 0=$ finite system of inequalities

$\mathrm{J}=$ index set of the inequalities

$£=$ infeasibility tolerance $€[0,1]$

We require that $\mathrm{hj}(\mathrm{x}, z) \geq 0$ (or $\mathrm{h}(\mathrm{x}, z) \geq 0)$ shall hold at least with some prescribed probability $1-£ \in[0,1]$, rather than for all possible realizations of $z$.

Mathmetical formulation: in this section, we first study the optimization problem of bandwidth assignment with a separate chance constraint for every microwave link and afterwards the joint chance constrained model. We assume in both models that, for each link and bandwidth, the modulation choice is associated with a random variable whose discrete probability distribution is known. In Figure(1) Let $£=0.05$ be the infeasibility tolerance (which corresponds to a reliability level of 0.95 ).

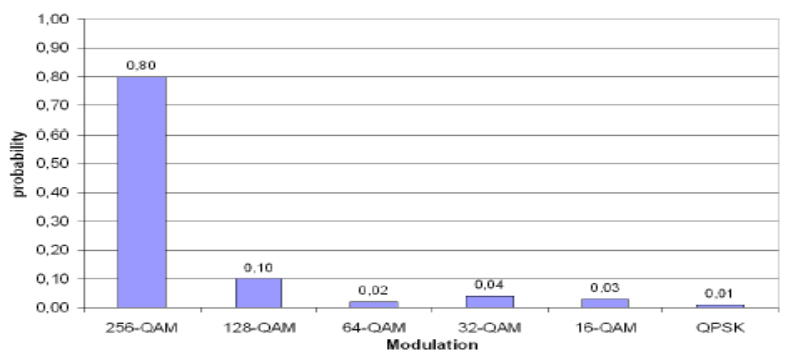

Fig (1) Discrete probability distribution function.

We can easily calculate the expectation value of the random variable $h, \mathrm{E}(h)=0.80 \times 8+0.10 \times 7+$ $0.02 \times 6+0.04 \times 5+0.03 \times 4+0.01 \times 2=7.56$. Note that, if we use the expectation value for estimating the link's capacity, the bandwidth choice of $10 \mathrm{MHz}$ is sufficient to carry all the demand (since the estimated capacity will be $7.56 \times 10=75.6 \mathrm{Mbps})$. However, $\mathrm{P}(10 . h \geq 70)=\mathrm{P}(h \geq 7)=0.90<0.95$. Thus, this solution does not respect the infeasibility tolerance $£=0.05$.

\section{Computational Result On Bandwidth Efficient}

Given the absence of topology instances for microwave backhaul networks available in the literature, we have performed computational experiments on a grid network (available at A particular characteristic of grid networks is the huge number of paths between any pair of nodes. In addition, the regular structure of grid networks make it easier to perform comparisons between various solutions methods. We take into account the $5 \times 5$ grid instance ( 25 base transceiver stations and 80 directional microwave links, as illustrated in Figure(2) ) with 50 demands taken from the original paper.

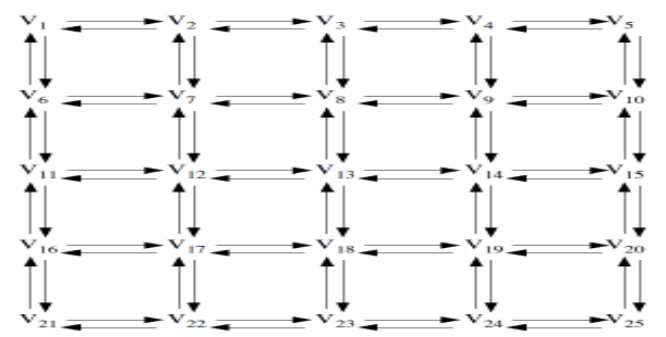

Fig(2) $5 \times 5$ network grid instance.

Price of reliability. As we assume the same availability for microwave links using the highest-level modulations and under the hypothesis that the lowest-level modulations are sufficiently robust to guarantee an availability of $100 \%$, instead of explicitly setting the infeasibility tolerance $£$, we can specify the maximum number of links $\mathrm{N}$ that we assume using highest-level modulations. 
As illustrated in Figure(3), the total bandwidth cost decreases as we admit larger values for the infeasibility tolerance ( $\mathrm{N}$ augments). For $\mathrm{N}=0$, when we have to assume the lowest-level modulations for all radio links, the network cannot provide enough capacity to satisfy all the traffic demands, hence this problem is infeasible. For $\mathrm{N}=10(£=0.01)$, the bandwidth cost is $38.6 \%$ higher than the bandwidth cost for $\mathrm{N}=80(£=$ 0.077 ) and $68.4 \%$ higher compared to the case where we do not use any optimization (i.e., we assign $28 \mathrm{MHz}$ for every microwave link). For $\mathrm{N}=60,70,80$, the probabilistic constraint does not really affect the cost of the solutions because the number of links we need to consider using the highest-level modulations to satisfy all the traffic requirement is smaller than 60 for these instances.

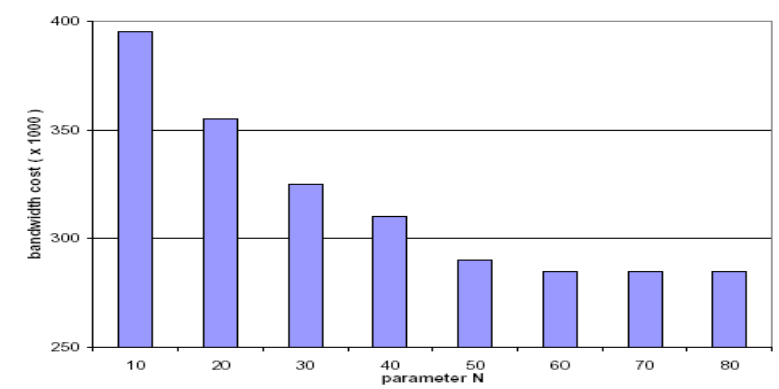

Fig(3) Bandwidth cost as a function of the infeasibility tolerance.

\section{Comparison Of The Formulation}

In addition, to study the gain of applying the valid inequalities introduced in we performed tests for different formulations A, B, C, D, that consider or not such valid inequalities according to the Table 1.

\begin{tabular}{llll}
\hline Formulation & Cutset & Type1 & Type2 \\
\hline A & no & no & no \\
B & yes & no & no \\
C & no & yes & yes \\
D & yes & yes & yes \\
\hline
\end{tabular}

Table 1 Different formulations w.r.t the valid inequalities.

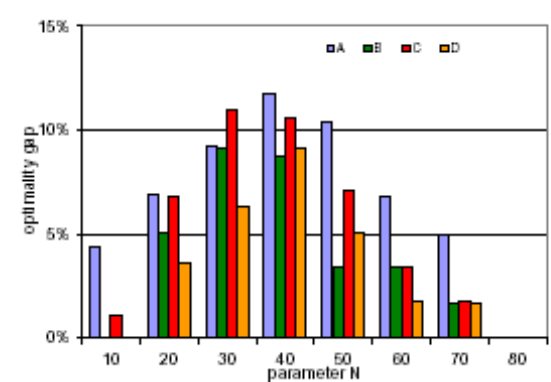

(a) Gap within nodes limit

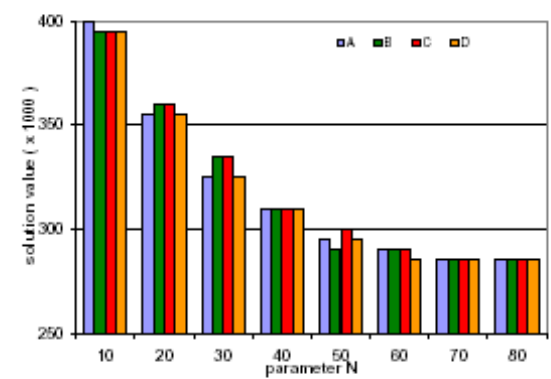

(c) Best solution

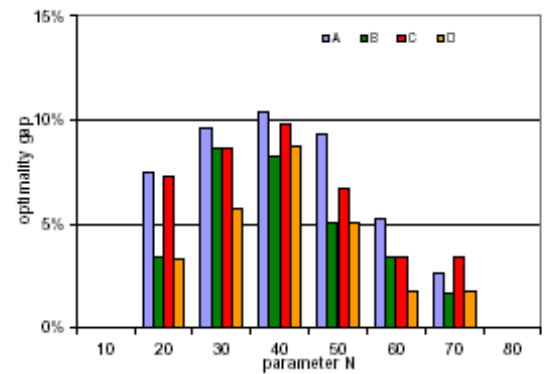

(b) Gap within time limit

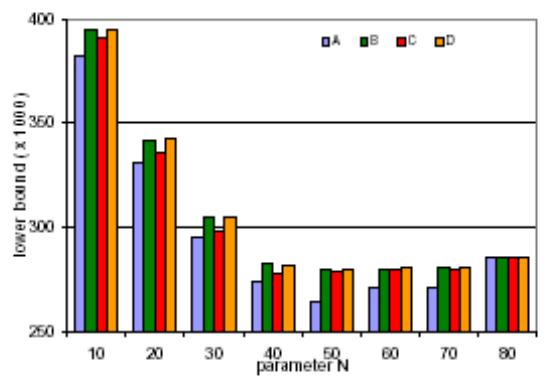

(d) Lower bound

Figure (4):Optimality gap, best solution and lower bound values achieved for the different formulations.

Figure illustrates, for each instance, the optimality gap (given by the ILP solver) achieved for the different formulations considering first a limit on the number of nodes (Figure (a)) and then on the execution 
time (Figure (b)), along with the best feasible solution (Figure (c)) and lower bound (Figure (d)) values obtained for the first scenario (limit on the number of nodes).

\section{Conclusion}

In this chapter, we have presented a chance-constrained programming approach to tackle the problem of assigning bandwidth for reliable microwave backhaul networks. We introduced different mathematical formulations and proposed cutset-based valid inequalities for this problem. In our computational studies, we discussed the price of reliability and compared the performance of the different mathematical

formulations. The results show that there is a tradeoff between bandwidth assignment costs and network reliability. In addition, the cutset-based valid inequalities improved the solving performance of the basic problem formulation. Many challenges related to this problem remain unaddressed. For example, besides the modulation scheme, we could consider other radio parameters (e.g., coding and transmission power) that vary in response to the conditions of the channel and that have a great impact on the capacity of a microwave link. Obviously, more realistic models while being more accurate also bring more difficulty on the problem solving.

\section{References}

[1]. K. Aardal, S. V. Hoesel, A. M. C. A. Koster, C. Mannino, and A. Sassano. Models and solution techniques for frequency assignment problems. Annals of Operations Research, 153(1):79-129, 2007.

[2]. G. Claßen, D. Coudert, A. M. C. A. Koster, and N. Nepomuceno. Bandwidth allocation for reliable fixed broadband wireless networks.

[3]. V. Gabrel, A. Knippel, and M. Minoux. Exact solution of multicommodity network optimization problems with general step cost functions.Operations Research Letters, 25(1):15-23, 1999.

[4]. V. Gabrel and M. Minoux. LP relaxations better than convexification for multicommodity network optimization problems with step increasing cost functions. Acta Mathematica Vietnamica, 22(1):123-145, 1997.

[5]. C. Raack, A. M. C. A. Koster, S. Orlowski, and R. Wessäly. On cutbased inequalities for capacitated network design polyhedra. Networks, 2010. 37, 38

[6]. F. Solano and M. Pióro. A mixed-integer programing formulation for the lightpath reconfiguration problem. In VIII Workshop on G/MPLS Networks (WGN8), 2009. 98, 99 